\title{
ON SOLUTIONS OF LINEAR DIFFERENTIAL EQUATIONS WITH REAL ZEROS; PROOF OF A CONJECTURE OF HELLERSTEIN AND ROSSI
}

\author{
FRANZ BRÜGGEMANN
}

(Communicated by Clifford J. Earle, Jr.)

\begin{abstract}
We prove the following conjecture that is due to Hellerstein and Rossi: Let $\left\{w_{1}, \ldots, w_{n}\right\}$ be a fundamental system of

$$
L w=w^{(n)}+a_{n-1}(z) w^{(n-1)}+\cdots+a_{0}(z) w \equiv 0
$$

with polynomials $a_{j}(z) \quad(0 \leq j \leq n-1)$. If each $w_{k}(1 \leq k \leq n)$ has only finitely many nonreal zeros, then there exists a polynomial $q(z)$ such that $u_{k}:=\exp (q(z)) w_{k} \quad(1 \leq k \leq n)$ form a fundamental system of a homogeneous linear differential equation with constant coefficients.
\end{abstract}

\section{INTRODUCTION}

In [7] Frank proved the following theorem:

Theorem 1. Let the linear differential equation $(D E)$

$$
L w=w^{(n)}+a_{n-1} w^{(n-1)}+\cdots+a_{0} w \equiv 0
$$

with $a_{j}(z)$ polynomials be given. Assume that there exists a fundamental system (FS) $\left\{w_{1}, \ldots, w_{n}\right\}$ of (1.1) with the property that each $w_{k}(1 \leq k \leq n)$ has only finitely many zeros. Then there exists a polynomial $q(z)$ such that $\widetilde{L} w:=\exp (-q) L(w \exp (q))$ is a linear differential polynomial with constant coefficients.

Recently, some investigations have been made in weakening the hypothesis of Theorem 1 that there exists a FS of (1.1) with finitely many zeros. One possibility is to allow infinitely many zeros under an additional condition on the geometric location of the zeros. More precisely, one can demand that all but finitely many zeros lie on a line, that can be taken as the real axis without loss of generality. In this direction we quote

Theorem 2 (Hellerstein, Shen, Williamson [10]). Let $w_{1}$ and $w_{2}$ be a FS of

$$
w^{\prime \prime}+P(z) w \equiv 0,
$$

Received by the editors December 29, 1989.

1980 Mathematics Subject Classification (1985 Revision). Primary 30D35, 34A20. 
where $P$ is a polynomial. If both $w_{1}$ and $w_{2}$ have only real zeros, then $P(z)$ is a constant.

Obviously, the conclusion of Theorem 1 remains true in the second order case under this weaker assumption. Hellerstein and Rossi asked whether an analogous result holds in the $n$th order case:

Conjecture (Brannan, Hayman [2, Problem 2.72, p. 13]). The conclusion of Theorem 1 remains true, if each $w_{k}(1 \leq k \leq n)$ has only finitely many nonreal zeros.

It is the purpose of this paper to prove the above conjecture. In fact, we will do this in a stronger form, by assuming that there are less nonreal than real zeros in a certain sense. More precisely, we will assume that for each $k \in\{1, \ldots, n\}$,

$$
\limsup _{r \rightarrow \infty} \log ^{+} n_{\mathrm{NR}}\left(r, w_{k}\right) / \log r<\rho\left(w_{k}\right) .
$$

Here $n_{\mathrm{NR}}\left(r, w_{k}\right)$ denotes the number of nonreal zeros (counted according to multiplicity) of $w_{k}$ in the circle $|z| \leq r$. Furthermore, $\rho\left(w_{k}\right)$ is the order of growth of $w_{k}$ that is defined by

$$
\rho\left(w_{k}\right)=\limsup _{r \rightarrow \infty} \log ^{+} \log ^{+} M\left(r, w_{k}\right) / \log r,
$$

where $M\left(r, w_{k}\right):=\max _{|z|=r}|w(z)|$ and $\log ^{+} r:=\max \{0, \log r\}$. If we replace $n_{\mathrm{NR}}\left(r, w_{k}\right)$ by the total number of zeros $n\left(r, w_{k}\right)$ and (1.3) holds with $n\left(r, w_{k}\right)$ instead of $n_{\mathrm{NR}}\left(r, w_{k}\right)$, then we say that $w_{k}$ has 0 as a Borel Exceptional Value (BEV). Gundersen [8] gave the following generalization of Theorem 2:

Theorem 3. Let $w_{1}, w_{2}$ be a FS of (1.2) with $\operatorname{deg}(P)=n$. If $n_{\mathrm{NR}}\left(r, w_{j}\right)=$ $o\left(r^{(n+2) / 2}\right)$ for $j=1,2$ as $r \rightarrow \infty$, then $P$ is a constant.

Note that for (1.2) $\rho\left(w_{1}\right)=\rho\left(w_{2}\right)=(n+2) / 2$. As a special case, Theorem 3 contains a result of Frank [7], who proved that the conclusion of Theorem 2 holds, if its assumption is replaced by: $w_{1}$ and $w_{2}$ have BEV 0 . In order to generalize Theorem 3 to the $n$th order case the following theorem, proved by the author $[3,4]$, is of interest:

Theorem 4. Let (1.1) be given with polynomial coefficients $a_{j}(z)(0 \leq j \leq n-1)$. Assume that there is a FS $\left\{w_{1}, \ldots, w_{n}\right\}$ such that $w_{k}$ either has $B E V 0$ or is a polynomial $(1 \leq k \leq n)$. Then either $a_{j}(z) \equiv$ const $(0 \leq j \leq n-1)$ or there exists a polynomial $q(z)$ with $w_{k}(z)=\exp (q(z)) u_{k}(z)$ and $\rho\left(u_{k}\right)<\operatorname{deg}(q)$.

We shall prove the following generalization of Theorem 3 to the $n$th order case, which implies Hellerstein and Rossi's conjecture.

Theorem 5. The conclusion of Theorem 4 holds, if the condition ' $w_{k}$ has BEV 0 ' is replaced by (1.3). Furthermore, if there is at least one transcendental $w_{k}$ 
with $\rho\left(w_{k}\right)=\max _{1 \leq j \leq n} \rho\left(w_{j}\right)$ that has not BEV 0 , then $a_{j}(z) \equiv$ const $\quad(0 \leq$ $j \leq n-1)$.

\section{TOOLS FROM ASYMPTOTIC INTEGRATION THEORY}

In order to prove Theorem 5, we make use of concepts from the theory of asymptotic integration that goes back to L. W. Thomé [13], H. Poincaré [12], and G. Birkhoff [1], and was recently refined by V. Dietrich [6]. From [6, 3], we know the existence of a formal FS of (1.1) with the following properties:

Theorem A. Let the DE (1.1) with rational functionals $a_{j}$ be given. There exists a formal $F S\left\{\tilde{w}_{1}, \ldots, \tilde{w}_{n}\right\}$ with

$$
\tilde{w}_{j}(z)=\exp \left(q_{j}\left(z^{1 / p}\right)\right) z^{\lambda_{j} / p}\left[\log \left(z^{1 / p}\right)\right]^{m_{j}} P_{j}\left(z^{1 / p}, \log \left(z^{1 / p}\right)\right)
$$

with polynomials $q_{j}, \lambda_{j} \in \mathbf{C}, m_{j} \in \mathbf{N}_{0}, p \in \mathbf{N} . P_{j}$ are rational functions in $\log \left(z^{1 / p}\right)$, whose coefficients are formal series of the form $\sum_{j \in \mathbf{N}_{0}} a_{j} z^{-j / p}$ that do not converge in general. $P_{j}$ has a formal asymptotic expansion of the form $P_{j}\left(z^{1 / p}, \log \left(z^{1 / p}\right)\right)=1+O\left(1 / \log \left(z^{1 / p}\right)\right)$. Furthermore, $\left(q_{j}, \lambda_{j}, m_{j}\right)$ are pairwise distinct and $m_{j}>0$ only occurs, if $\left(q_{j}, \lambda_{j}, k\right)\left(0 \leq k \leq m_{j}-1\right)$ also appear in (2.1).

It should be remarked that (according to Dietrich [5]) the terms $\left(q_{k}, \lambda_{k}, m_{k}\right)$ and $p$ can be computed from the coefficients of (1.1). As already pointed out, normally the series in (2.1) do not converge. However, there is a strong relationship between the actual solutions of (1.1) and the formal solutions (2.1). Concretely, the actual solutions are represented asymptotically by formal solutions in suitably chosen sectors. The width of these sectors depends on the exponential parts of (2.1). We need the following:

Definition (Stokes ray, normal sector). (a) Let the DE (1.1) and its formal FS (2.1) be given; $\gamma \in[0,2 \pi]$ is called a Stokes ray, if there are different $q_{j}, q_{k}$ with $\operatorname{deg}\left(q_{j}-q_{k}\right)=l$ and $\varepsilon>0$ such that

$$
\lim _{\substack{|z| \rightarrow \infty \\ \arg z=\varphi}} \frac{\operatorname{Re}\left[q_{j}\left(z^{1 / p}\right)-q_{k}\left(z^{1 / p}\right)\right]}{|z|^{l / p}} \begin{cases}>0, & \varphi \in(\gamma, \gamma+\varepsilon) \\ <0, & \varphi \in(\gamma-\varepsilon, \gamma) .\end{cases}
$$

We get finitely many Stokes rays and numerate them with respect to growth

$$
\gamma_{1}<\gamma_{2}<\cdots<\gamma_{m} \quad\left(m \in \mathbf{N}_{0}\right) \text {. }
$$

If $m=0$, then no Stokes ray exists; that is, $q_{j}=q$ for all $j$ in (2.1).

(b) Given (2.3) with $m>0$ we denote the $\mu$ th normal sector of $(1.1)$ as

$$
S_{\mu}:=\left\{z \in \mathbf{C} \mid \gamma_{\mu-1}<\arg z<\gamma_{\mu+1}\right\} \quad(1 \leq \mu \leq m) \text {. }
$$

Here we take $\gamma_{0}:=\gamma_{m}-2 \pi$ and $\gamma_{m+1}:=\gamma_{1}+2 \pi$. If $m=0$, then $S_{0}:=\mathbf{C}$. We will also need

$$
\begin{aligned}
& S_{\mu}^{+}(\varepsilon):=\left\{z \in \mathbf{C} \mid \gamma_{\mu}+\varepsilon \leq \arg z \leq \gamma_{\mu+1}-\varepsilon\right\}, \\
& S_{\mu}^{-}(\varepsilon):=\left\{z \in \mathbf{C} \mid \gamma_{\mu-1}+\varepsilon \leq \arg z \leq \gamma_{\mu}-\varepsilon\right\} .
\end{aligned}
$$


Note that the normal sector $S_{\mu}$ contains the Stokes ray $\gamma_{\mu}$. The following theorem is the main result of asymptotic integration theory:

Theorem B. Let (1.1) and (2.1) be given. Then for each normal sector $S_{\mu}$ there exists a $F S\left\{y_{1}, \ldots, y_{n}\right\}$ of (1.1) with

$$
y_{j}(z)=\exp \left(q_{j}\left(z^{1 / p}\right)\right) z^{\lambda_{j} / p}\left(\log \left(z^{1 / p}\right)\right)^{m_{j}}(1+o(1)) ; \quad\left(|z| \rightarrow \infty, \quad z \in S_{\mu}\right) .
$$

To get a simpler formulation, we say that ' $y_{j}$ is asymptotically represented by $\left(q_{j}, \lambda_{j}, m_{j}\right)$ in $S_{\mu}$ ' if $(2.6)$ holds in $S_{\mu}$. Of course, the asymptotic relation (2.6) can be refined, but (2.6) is sufficient for our purposes. We now describe the connection between the indicator function of a solution $w(z)$ of $(1.1)$ and the formal indicator functions. To do this, let $J\left(S_{\mu}, w\right) \subset\{1, \ldots, n\}$ be the set of integers $j$, such that $c_{j} \neq 0$ in $w(z)=\sum_{j=1}^{n} c_{j} y_{j}(z)$, where $y_{j}$ represents $\left(q_{j}, \lambda_{j}, m_{j}\right)$ asymptotically in $S_{\mu}$.

For $q_{j}\left(z^{1 / p}\right)=a_{j} z^{l_{j} / p}(1+o(1)) ; a_{j} \in \mathbf{C}, l_{j} \in \mathbf{N}, a_{j}=0$ only if $q_{j} \equiv 0$, we define the formal indicator function:

$$
I_{j}(\vartheta):=\left|a_{j}\right| \cos \left(\arg \left(a_{j}\right)+\vartheta l_{j} / p\right) \quad(1 \leq j \leq n) .
$$

Let $w(z)$ be a solution of (1.1). As the coefficients of (1.1) are polynomials, $w(z)$ is an entire function of finite order of growth (compare e.g. [11, p. 180]). If $\rho(w)>0$, the indicator function $h_{w}(\vartheta)$ of $w$, which is $2 \pi$-periodic, is given as

$$
h_{w}(\vartheta):=\limsup _{r \rightarrow \infty} \log \left|w\left(r e^{i \vartheta}\right)\right| / r^{\rho(w)} \quad(\vartheta \in \mathbf{R}) .
$$

Furthermore, we define

$$
\widehat{I}_{j}(\vartheta, w):= \begin{cases}I_{j}(\vartheta), & \text { if } \rho(w)=l_{j} / p \\ 0, & \text { otherwise } .\end{cases}
$$

Now we are able to define $I(\vartheta, w):[0,2 \pi] \rightarrow \mathbf{R}$ by

$$
I(\vartheta, w):=\max \left\{\hat{I}_{j}(\vartheta, w) \mid j \in J\left(S_{\mu}, w\right)\right\} \text { for all } \vartheta \in\left(\gamma_{\mu-1}, \gamma_{\mu+1}\right) .
$$

Of course, the intervals $\left(\gamma_{\mu-1}, \gamma_{\mu+1}\right)$ overlap. However, it is easy to show that $I(\vartheta, w)$ is well defined [6]. In [6] Dietrich proved the following important

Theorem C. Let $w(z)$ be a solution of (1.1) with $\rho(w)>0$. Then $h_{w}(\vartheta)=$ $I(\vartheta, w)$ for all $\vartheta \in[0,2 \pi]$.

\section{Proof of Theorem 5}

First we introduce the following notation: we say that a solution $w(z)$ of (1.1) of order $\rho(w)>0$ has an essential asymptotic change at $\gamma \in[0,2 \pi]$, if $\gamma$ is a Stokes ray and the polynomials $q_{j}, q_{k}$ in (2.2) are of the form $q_{j}\left(z^{1 / p}\right)=a_{j} z^{\rho(w)}+O\left(z^{\rho(w)-1 / p}\right) ; \quad q_{k}\left(z^{1 / p}\right)=a_{k} z^{\rho(w)}+O\left(z^{\rho(w)-1 / p}\right)$ with $a_{j} \neq a_{k}$; furthermore it is demanded that $h_{w}(\vartheta)=\widehat{I}_{j}(\vartheta, w)$ for $\vartheta \in(\gamma, \gamma+c)$ 
and $h_{w}(\vartheta)=\widehat{I}_{k}(\vartheta, w)$ for $\vartheta \in(\gamma-c, \gamma)$ for a suitable $c>0$. Moreover, we say that $w(z)$ has essentially many zeros in a sector $S$, if the number $n_{S}(r, w)$ of zeros in $S \cap\{z|| z \mid \leq r\}$ is of the form $n_{S}(r, w)=\kappa r^{\rho(w)}(1+o(1)) \quad(r \rightarrow \infty)$ for a $\kappa>0$. Then we have

Lemma 1. For a given ray $\vartheta \in[0,2 \pi]$, a solution $w(z)$ of $(1.1)$ of order $\rho(w)>0$ has essentially many zeros in each arbitrary small sector $S$ containing the ray $\arg z=\vartheta$, if and only if $w(z)$ has an essential asymptotic change at $\vartheta$.

Proof. The conclusion is an easy consequence of results from the value distribution theory of exponential polynomials (compare e.g. H. L. Turrittin [14]). From Theorem $\mathrm{B}$ we deduce that in an appropriate sector $w(z)$ is represented by a slightly disturbed exponential polynomial and, as pointed out in [3], one can transfer these results of exponential polynomials to solutions of the DE (1.1). Hence it is clear that essentially many zeros can only occur in arbitrarily small sectors containing those Stokes rays (2.2) that indicate an essential asymptotic change of $w(z)$.

The assumption of Theorem 5 means that an essential asymptotic change for $w_{k}(1 \leq k \leq n)$ can only take place at the positive or negative real axis $(\gamma=0$ or $\gamma=\pi$ ). Next we show

Lemma 2. $\rho\left(w_{l}\right)$ is an integer for $1 \leq l \leq n$.

Proof. Suppose that $\rho\left(w_{l}\right)=\rho$. If $w_{l}$ has no essential asymptotic change, then $w_{l}$ possesses a fixed leading exponential part and therefore it has BEV 0 , which implies (e.g. [11, p. 123]) that $\rho$ is an integer. Otherwise $w_{1}$ has exactly two essential asymptotic changes, namely at $\gamma=0$ and at $\gamma=\pi$. Clearly there are two formal indicator functions $I_{j_{1}}(\vartheta) \neq I_{j_{2}}(\vartheta)$ such that (note Theorem C)

$$
\begin{aligned}
& \widehat{I}_{j_{1}}\left(\vartheta, w_{l}\right)=h_{w_{l}}(\vartheta) \quad(\vartheta \in[0, \pi]), \\
& \widehat{I}_{j_{2}}\left(\vartheta, w_{l}\right)=h_{w_{l}}(\vartheta) \quad(\vartheta \in[\pi, 2 \pi]) .
\end{aligned}
$$

If, for example, $\widehat{I}_{j_{1}}\left(\vartheta, w_{l}\right) \equiv 0$, then clearly $\widehat{I}_{j_{2}}\left(0, w_{l}\right)=\widehat{I}_{j_{2}}\left(\pi, w_{l}\right)=0$. As $\widehat{I}_{j_{2}}\left(\vartheta, w_{l}\right)=\left|a_{j_{2}}\right| \cos \left(\arg \left(a_{j_{2}}\right)+\rho \vartheta\right)$, this implies that $\rho$ is an integer. If both $\widehat{I}_{j_{1}}$ and $\widehat{I}_{j_{2}}$ do not vanish identically, we have

$$
\begin{aligned}
\left|a_{j_{1}}\right| \cos \left(\arg \left(a_{j_{1}}\right)\right) & =\left|a_{j_{2}}\right| \cos \left(\arg \left(a_{j_{2}}\right)\right), \\
\left|a_{j_{1}}\right| \cos \left(\arg \left(a_{j_{1}}\right)+\rho \pi\right) & =\left|a_{j_{2}}\right| \cos \left(\arg \left(a_{j_{2}}\right)+\rho \pi\right),
\end{aligned}
$$

and from the last equation we deduce that

$$
\left|a_{j_{1}}\right| \sin \left(\arg \left(a_{j_{1}}\right)\right) \sin (\rho \pi)=\left|a_{j_{2}}\right| \sin \left(\arg \left(a_{j_{2}}\right)\right) \sin (\rho \pi) .
$$

Now either $\rho$ is an integer or $a_{j_{1}}=a_{j_{2}}$, contradicting our assumption.

From now on we shall assume that $\rho\left(w_{l}\right)=\rho(1 \leq l \leq n)$. This is no restriction, because otherwise in view of Lemma 2 we could make a suitable transformation $u:=w \exp \left(c z^{\rho}\right)$ for appropriate $c \in \mathbf{C} \backslash\{\mathbf{0}\}$ and $\rho=\max _{1 \leq l \leq n} \rho\left(w_{l}\right)$. 
Note that this transformation has no influence on the zeros of $w_{l}(1 \leq l \leq n)$. With respect to $h_{w_{l}}(\vartheta)$ the following holds:

Lemma 3. Consider the special FS $\left\{w_{1}, \ldots, w_{n}\right\}$ of Theorem 5. Given $w_{k}$ there either exists $j \in\{1, \ldots, n\}$ with

$$
h_{w_{l}}(\vartheta)=I_{j}(\vartheta)=\left|a_{j}\right| \cos \left(\arg \left(a_{j}\right)+\rho \vartheta\right) \quad(\vartheta \in[0,2 \pi])
$$

or there exist $I_{j_{1}} \neq I_{j_{2}}$ such that

$$
\begin{array}{ll}
h_{w_{l}}(\vartheta)=I_{j_{1}}(\vartheta) & (\vartheta \in[0, \pi]), \\
h_{w_{l}}(\vartheta)=I_{j_{2}}(\vartheta) & (\vartheta \in[\pi, 2 \pi]) .
\end{array}
$$

In the first case $h_{w_{l}}(\vartheta)$ has the period $2 \pi / \rho$, whereas in the second case $h_{w_{l}}(\vartheta)$ has this period restricted to the upper (respectively lower) halfplane.

The proof of Lemma 3 is an easy consequence of (3.1). In view of (3.4) and (3.5) we shall divide the FS $\left\{w_{1}, \ldots, w_{n}\right\}$ into subsets $M_{1}:=\left\{w_{1}, \ldots, w_{r}\right\}$ and $M_{2}:=\left\{w_{r+1}, \ldots, w_{n}\right\}$ such that the elements of $M_{1}$ fulfill (3.4) and those of $M_{2}$ (3.5). If $M_{2}=\varnothing$, then Theorem 4 can be applied directly and the conclusion follows. Hence we may assume that $M_{2} \neq \varnothing$. We proceed to prove

Lemma 4. If $M_{2} \neq \varnothing$, then $\rho=1$.

Proof. Assume that $\rho>1$. According to (3.5) we write $I_{j_{1}}^{(l)}, I_{j_{2}}^{(l)}(r+1 \leq$ $l \leq n)$ for the formal indicator functions representing $h_{w_{l}}$ in the upper (lower) halfplane. Let $\gamma_{\mu}$ denote the Stokes ray $\arg z=\pi$. Then for arbitrarily small $\varepsilon$ there is a formal indicator function $I_{\kappa}$, that, restricted to $S_{\mu}^{-}(\varepsilon)$, is maximal in $\left\{I_{j_{1}}^{(l)} \mid r+1 \leq l \leq n\right\}$. Now $I_{\kappa} \notin\left\{I_{j_{2}}^{(l)} \mid r+1 \leq l \leq n\right\}$. Otherwise, there would exist $I_{\tilde{\kappa}} \in\left\{I_{j_{1}}^{(l)} \mid r+1 \leq l \leq n\right\}$ and $w_{l} \in M_{2}$ with $I_{j_{1}}^{(l)}=I_{\tilde{\kappa}}, I_{j_{2}}^{(l)}=I_{\kappa}$, contradicting (according to (2.10)) the maximality of $I_{\kappa}$ in $S_{\mu}^{-}(\varepsilon)$.

We now show that $\rho=2$ is impossible. To do this, we denote the normal sector containing the Stokes ray $\arg z=0$ by $S_{\tilde{\mu}}$. According to (3.5), for a given $l \in\{r+1, \ldots, n\}$, we get

$$
\begin{array}{ll}
I_{j_{1}}^{(l)}>I_{j_{2}}^{(l)} & \text { in } S_{\mu}^{-}(\varepsilon) \cup S_{\tilde{\mu}}^{+}(\varepsilon), \\
I_{j_{1}}^{(l)}<I_{j_{2}}^{(l)} & \text { in } S_{\mu}^{+}(\varepsilon) \cup S_{\tilde{\mu}}^{-}(\varepsilon) .
\end{array}
$$

Now, if $\rho=2, I_{j_{1}}^{(l)}$ and $I_{j_{2}}^{(l)}$ have the period $\pi$ and, therefore, $I_{j_{1}}^{(l)}>I_{j_{2}}^{(l)}$ in $S_{\mu}^{-}(\varepsilon) \cup S_{\tilde{\mu}}^{-}(\varepsilon)$ contradicting (3.6). Hence $\rho \geq 3$.

For $I_{\kappa}(\vartheta)$ we have a corresponding leading exponential part $\exp \left(c z^{\rho}\right), c \in$ $\mathbf{C} \backslash\{\mathbf{0}\}$. Assume further that $M_{1}$ contains $s$ solutions with fixed leading exponential part $\exp \left(c z^{\rho}\right)$. Let $\tau$ denote the number of formal solutions (2.1) with 
leading $\exp \left(c z^{\rho}\right)$. We shall investigate two cases:

(i) $s<\tau$. Consider the Stokes ray $\gamma_{\tilde{\mu}}=\pi+2 \pi / \rho$. As $\rho \geq 3$, we have $\gamma_{\tilde{\mu}}<2 \pi$. Because of the maximality of $I_{\kappa}$ in $S_{\mu}^{-}(\varepsilon)$ and its $2 \pi / \rho$-periodicity we obtain

$$
h_{w_{l}}(\vartheta)<I_{\kappa}(\vartheta) \quad\left(\vartheta \in\left(\gamma_{\tilde{\mu}-1}+\varepsilon, \gamma_{\tilde{\mu}}+\varepsilon\right)\right),(r+1 \leq l \leq n) .
$$

In view of (3.7) it is impossible to generate $\tau$ functions $y_{l}(1 \leq l \leq \tau) \in$ $\operatorname{span}\left\{w_{1}, \ldots, w_{n}\right\}$, that have leading exponential part $\exp \left(c z^{\rho}\right)$ and represent different $\left(q_{l}, \lambda_{l}, m_{l}\right)$ asymptotically in $S_{\tilde{\mu}}(\varepsilon)$. Obviously this contradicts Theorem B.

(ii) $s=\tau$. Consider the Stokes ray $\gamma_{\tilde{\mu}}=\pi-\pi / \rho$. Clearly for all $j$ : $I_{j}(\vartheta-\pi / \rho)=-I_{j}(\vartheta)$. So $I_{\kappa}$, restricted to $S_{\tilde{\mu}}^{-}(\varepsilon)$, is minimal in $\left\{I_{j_{1}}^{(l)} \mid r+1 \leq\right.$ $l \leq n\}$. Let $\nu$ denote the number of solutions $w_{l} \in M_{1}$ with $h_{w_{l}}<I_{\kappa}$ in $S_{\tilde{\mu}}^{-}(\varepsilon)$. We show that there are exactly $\nu$ solutions $y_{l}$ of (3) with $h_{y_{l}}<I_{\kappa}$ in this sector: suppose that there are $t>\nu$ solutions $y_{l}$ with this property. Then there exists a FS $\left\{\tilde{y}_{1}, \ldots, \tilde{y}_{n}\right\}$ having the asymptotic expansions $(3)$ in $S_{\mu}^{-}(\varepsilon)$ and at least $t$ solutions have an indicator function $>I_{\kappa}$. Because of the maximality of $I_{\kappa}$ in $\left\{I_{j_{1}}^{(l)} \mid l \in M_{2}\right\}$ (restricted to $S_{\mu}^{-}(\varepsilon)$ ) there are exactly $t$ solutions $w_{l} \in M_{1}$ with the property $h_{w_{l}}>I_{\kappa}$ restricted to $S_{\mu}^{-}(\varepsilon)$ and hence $h_{w_{l}}<I_{\kappa}$ restricted to $S_{\tilde{\mu}}^{-}(\varepsilon)$. This is an obvious contradiction. So there exist $n-\nu-\tau$ solutions $y_{l}$ with different asymptotic $\left(q_{l}, \lambda_{l}, m_{l}\right)$ and the property $h_{y_{l}}>I_{\kappa}$ in $S_{\tilde{\mu}}^{-}(\varepsilon)$. However $\left\{w_{1}, \ldots, w_{n}\right\}$ contains at least $\nu+\tau+1$ solutions $w_{l}$ with $h_{w_{l}} \leq I_{\kappa}$, so that $\left\{y_{1}, \ldots, y_{n}\right\}$ cannot be generated by $\left\{w_{1}, \ldots, w_{n}\right\}$. This completes the proof of Lemma 4.

Now the proof of Theorem 5 follows from Lemma 4 and the well-known result [15] that $\rho\left(w_{l}\right) \leq 1 \quad(1 \leq l \leq n)$ implies constant coefficients in the case of polynomial coefficients in (1.1).

\section{Proof of the Hellerstein-Rossi conjecture}

It remains to be shown that Theorem 5 implies Hellerstein's and Rossi's conjecture. Clearly the conjecture immediately follows if one of the solutions $w_{k}$ does not have 0 as a BEV. If all $w_{k}(1 \leq k \leq n)$ have $\mathrm{BEV} 0$, then in view of Theorem 4 the order $\rho$ of the solutions can be reduced by a suitable transformation $u_{k}(z):=\exp \left(c z^{\rho}\right) w_{k}(z)$ with $\rho\left(u_{k}\right)<\rho(1 \leq k \leq n)$. Furthermore, the hypothesis on the zeros also holds for the $u_{k}$. Hence we can apply Theorem 5 to the DE corresponding to $\left\{u_{1}, \ldots, u_{n}\right\}$. If we continue this process we finally arrive at $\rho=1$ and so there is a polynomial $P(z)$ such that $w_{k}(z)=\exp (P(z)) v_{k}(z)$ with $\rho\left(v_{k}\right) \in\{0,1\}$. Therefore (1.1) can be transformed to a DE with constant coefficients. 


\section{SOME REMARKS}

(a) To avoid a too pompous formulation, we demanded that most of the zeros lie exactly on the real axis. Of course, all the results hold if we replace this by 'most of the zeros lie in arbitrarily small sectors around the positive and negative real axis.'

(b) In [9] Hellerstein and Rossi proved

Theorem 6. Let (1.2) with a rational function $P(z)$ be given. Suppose further that there exist two linear independent meromorphic solutions $w_{1}, w_{2}$ satisfying (1.3). Furthermore, assume that $w_{1} w_{2}$ is transcendental. Then $P(z)=a+$ $O(1 / z),(|z| \rightarrow \infty)$ with $a \in \mathbf{C} \backslash\{\mathbf{0}\}$.

Theorem 6 can be transferred to the $n$th order case:

Theorem 7. Assume that (1.1) is given with rational coefficients $a_{j}$. Suppose that there exists a FS of meromorphic solutions $w_{k}(1 \leq k \leq n)$ satisfying (1.3). Moreover, assume that at least one $w_{k}$ of maximal order does not have 0 as a $B E V$. Then $a_{j}(z)=\alpha_{j}+O(1 / z) \quad(|z| \rightarrow \infty, \alpha \in \mathbf{C})$ and there is at least one $j$ with $\alpha_{j} \neq 0$.

Proof. Obviously, the method of asymptotic integration can be applied again. Analogously to the proof of Theorem 5 we derive $\rho\left(w_{k}\right) \in\{0,1\} \quad(1 \leq k \leq n)$ and there is at least one $k$ with $\rho\left(w_{k}\right)=1$. This implies that the NewtonPuiseux diagram (e.g. [5]) of the DE presents a single line with NP-slope 1, that directly gives the proclaimed representation of $a_{j}(z)$.

\section{REFERENCES}

1. G. D. Birkhoff, Singular points of ordinary linear differential equations, Trans. Amer. Math. Soc. 10 (1909), 436-470.

2. D. A. Brannan and W. K. Hayman, Research problems in complex analysis, Bull. London Math. Soc. 21 (1989), 1-35.

3. F. Brüggemann, Untersuchungen linearer Differentialgleichungen mit rationalen Koeffizienten im Komplexen, Dissertation, RWTH, Aachen, 1989.

4. _ On the zeros of fundamental systems of linear differential equations with polynomial coefficients, Complex Variables Theory Appl. (to appear).

5. V. Dietrich, Newton-Puiseux diagramm für Systeme linearer Differentialgleichungen, Complex Variables Theory Appl. 7 (1987), 256-296.

6. __ Über die Annahme der möglichen Wachstumsordnungen und Typen bei linearen Differentialgleichungen, Habilitationsschrift, RWTH, Aachen, 1989.

7. G. Frank, Picardsche Ausnahmewerte bei Lösungen linearer Differentialgleichungen, Manuscripta Math. 2 (1970), 181-190.

8. G. Gundersen, On the real zeros of solutions to $f^{\prime \prime}+A f=0$ where $f$ is entire, Ann. Acad. Sci. Fenn. Ser. A I Math. 11 (1986), 275-294.

9. S. Hellerstein and J. Rossi, Zeros of meromorphic solutions of second order linear differential equations, Math. Z. 192 (1986), 603-612.

10. S. Hellerstein, L. C. Shen, and J. Williamson, Real zeros of derivatives of meromorphic functions and solutions of second-order differential equations, Trans. Amer. Math. Soc. 285 (1984), 759-776. 
11. G. Jank and L. Volkmann, Einführung in die Theorie der ganzen und meromorphen Funktionen mit Anwendungen auf Differentialgleichungen, Birkhäuser, Basel, Boston, and Stuttgart, 1985.

12. H. Poincaré, Sur les intégrales des équations linéaires, Acta Math. 8 (1886), 295-344.

13. L. W. Thomé, Zur Theorie der linearen Differentialgleichungen, J. Reine Angew. Math. 75 (1873), 265-291.

14. H. L. Turrittin, Asymptotic distribution of zeros for certain exponential sums, Amer. J. Math. 66 (1944), 199-228.

15. H. Wittich, Zur Kennzeichnung linearer Differentialgleichungen mit konstanten Koeffizienten, Festband zum 70. Geburtstag von Rolf Nevanlinna (H. P. Künzi and A. Pfluger, eds.), Springer-Verlag, 1966.

Lehrstuhl II für Mathematik, RWTH Aachen, Templergraben 55, D-5100 Aachen, FedERAL REPUBLIC OF GERMANY 